\title{
Agente espumante e seus efeitos nas propriedades físicas de alumina porosa
}

\author{
(Foaming agent and the effects on the physical \\ properties of porous alumina)
}

\author{
V. R. Salvini, J. R. Garcia, I. R. de Oliveira, V. C. Pandolfelli \\ Departamento de Engenharia de Materiais - DEMa \\ Universidade Federal de S. Carlos - UFSCar \\ Rod. Washington Luiz, Km 235, C.P. 676, S. Carlos, SP 13565-905 \\ pvrs@iris.ufscar.br,vicpando@power.ufscar.br
}

\begin{abstract}
Resumo
Cerâmicas porosas têm sido aplicadas em processos de refino, microfiltração de líquidos e emulsões e, também, como isolantes térmicos a altas temperaturas. Embora exista uma vasta aplicação tecnológica destes materiais, há poucos estudos na literatura sobre a obtenção de cerâmicas usando-se espuma como precursora da porosidade e seus efeitos nas propriedades finais do corpo. Neste contexto, o objetivo do presente trabalho compreende a avaliação de distintos agentes químicos espumantes catiônicos e aniônicos e de um agente viscosificante na produção e estabilidade de espumas aquosas. Os critérios de avaliação destes aditivos foram o volume de espuma produzido, o tempo para colapsar as bolhas de ar e a natureza química dos surfactantes. Os corpos cerâmicos porosos obtidos foram avaliados quanto à sua porosidade, densidade e distribuição de tamanho de poros.
\end{abstract}

Palavras-chave: espumas, alumina porosa, processamento cerâmico.

\begin{abstract}
Porous ceramics have been applied in the refining process, micro filtration of liquids and emulsions and, also, as thermal insulators for high temperatures. Nevertheless, there are few studies in the literature concerning the production of ceramics using foam as the porosity precursor and its effect on the final properties of the material. Thus, the objective of this work comprises the evaluation of the different cationic and anionic foaming chemical additives, and a thickening agent on the production and stabilization of the water based foams. The evaluating criteria of these agents involved the foam volume generated, the time for the coalescence of the air bubbles and the chemical nature of the surfactants. The properties of the porous ceramic samples were carried out considering the porosity, the density and the distribution of pore size.
\end{abstract}

Keywords: foams, porous alumina, ceramic processing.

\section{INTRODUÇÃO}

Cerâmicas porosas têm sido utilizadas em diversas aplicações tecnológicas, tais como coletores de partículas sólidas, isolantes térmicos para altas temperaturas, isolantes acústicos e como queimadores de materiais [1-4].

Estes materiais podem ser produzidos pela adição de matérias-primas de baixa densidade, tais como o uso de fibras poliméricas, esferas ocas e argila expandida, ou pela incorporação de gases à mistura cerâmica e/ou pela combinação destas técnicas[1-4]. As principais diferenças entre estes métodos estão relacionadas ao custo, toxidade e refratariedade das matérias-primas utilizadas, além da distribuição de tamanho de poros formados.

A incorporação de ar à mistura cerâmica apresenta algumas vantagens na obtenção de cerâmicas porosas refratárias, tais como o baixo custo e o uso de aditivos atóxicos.

Fundamentalmente, há duas maneiras de se incorporar ar ou outros gases à mistura cerâmica. A primeira é pela geração de um gás in situ como resultado de uma reação química. Como exemplo, tem-se a liberação de hidrogênio pela reação da água com o pó de alumínio metálico, resultando numa estrutura com poros na forma capilar.

Aoutra maneira compreende a incorporação de ar por agitação ou adição da espuma aquosa à suspensão cerâmica. Neste caso a estrutura resultante apresenta poros esféricos $[5,6]$.

Uma vez que pela agitação da suspensão não se tem o controle do tamanho da bolha de ar e da porosidade [5], a adição da espuma aquosa tem-se mostrado um método eficiente na obtenção de corpos de alta porosidade $(>70 \%)$ e tamanho de poros pequenos $(<10 \mu \mathrm{m})[6]$. 
No presente trabalho adotou-se a técnica de incorporação da espuma aquosa para a fabricação dos corpos cerâmicos porosos. $\mathrm{Na}$ aplicação desta técnica, geralmente as espumas aquosas são formadas separadamente por meio da mistura de soluções de agentes surfactantes e, então, adicionadas à suspensão cerâmica [7]. Os agentes surfactantes são os responsáveis pela formação da espuma atuando na diminuição da tensão superficial da solução, o que favorece o surgimento da interface água-ar [8]. Os agentes surfactantes podem ter diferentes estruturas químicas, entretanto os surfactantes iônicos (aniônicos e catiônicos) são os que apresentam características mais indicadas para a produção de espumas estáveis [9], qualidade imprescindível para que esta possa ser incorporada ao material cerâmico.

Neste contexto, o objetivo deste trabalho é estudar o efeito de diferentes agentes surfactantes na produção e na estabilidade das espumas aquosas e sua conseqüente influência nas propriedades de corpos cerâmicos porosos. Para isto, foram avaliados surfactantes catiônicos e aniônicos. Os critérios de avaliação destes surfactantes foram o volume de espuma produzida e o tempo necessário para drenagem da fase líquida. Os corpos cerâmicos porosos obtidos foram avaliados quanto à sua porosidade, densidade e distribuição de tamanho de poros.

\section{MATERIAIS E MÉTODOS}

\section{Materiais}

Inicialmente as suspensões cerâmicas aquosas foram preparadas com a alumina $\mathrm{Al}_{2} \mathrm{O}_{3}$ CT $3000 \mathrm{SG}$ e 5\%-p de cimento CA-14, ambos da Almatis (EUA). O aditivo citrato de diamônio (DAC, Sigma) foi utilizado como dispersante.

Paralelamente, prepararam-se as espumas em um agitador mecânico utilizando uma hélice helicoidal.

Para a produção das espumas aquosas foram selecionados quatro surfactantes, sendo dois deles catiônicos (um quaternário de amônio com cadeia alquílica linear e outro com cadeia ramificada) e dois aniônicos (uma olefina sulfonada e um alquil benzeno sulfonado).

Com o objetivo de se aumentar a estabilidade das espumas testadas, estudou-se o efeito da adição de um agente viscosificante no volume de espuma gerado e no tempo de estabilidade desta espuma. A presença de um agente viscosificante minimiza a ação de mecanismos de degradação da espuma, tais como a drenagem gravitacional e sucção capilar [9], que tem sua ação diminuída com o aumento da viscosidade da fase líquida. Os agentes viscosificantes mais utilizados nestes casos são polímeros hidrossolúveis $[8,9]$, sendo que neste trabalho utilizou-se um composto a base de celulose.

Após a homogeneização da suspensão cerâmica com a espuma aquosa, corpos cilíndricos foram moldados e curados à $25{ }^{\circ} \mathrm{C} / 24 \mathrm{~h}$. Em seguida, estes corpos foram desmoldados, secos a $100{ }^{\circ} \mathrm{C}$ por $12 \mathrm{~h}$ e, finalmente, sinterizados a $1100{ }^{\circ} \mathrm{C} / 1 \mathrm{~h}$ ao ar.

\section{Métodos}

A eficiência dos surfactantes como agente espumante foi avaliada quanto ao volume de espuma gerada após 3 min de aeração em um agitador mecânico utilizando-se uma ferramenta helicoidal. Estas medidas foram realizadas com a quantidade de $50 \mathrm{~mL}$ de soluções dos surfactantes com valores de concentração variando entre $0,2 \mathrm{~g} / \mathrm{L}$ a $100 \mathrm{~g} / \mathrm{L}$.

Já a avaliação quanto a estabilidade das espumas consistiu na medida do tempo necessário para a drenagem de metade do volume inicial de solução $(25 \mathrm{~mL})$, imediatamente após a formação da espuma. Para isto, preparou-se a espuma conforme anteriormente descrito em uma proveta graduada de $1000 \mathrm{~mL}$. Em seguida, acompanhou-se a formação do líquido na base da proveta.

As propriedades físicas das amostras sinterizadas foram avaliadas quanto às porosidades total, aparente e fechada, densidade aparente e relativa e distribuição do tamanho de poros.

A densidade relativa foi calculada segundo a expressão:

$$
\rho_{\text {relativa }}=\frac{\rho_{\mathrm{v}}}{\rho_{\text {real }}}
$$

onde $\rho_{\mathrm{v}}$ é a densidade volumétrica e $\rho_{\text {real }}$, é a densidade real da cerâmica efetuada após queima e moagem da cerâmica porosa no Picnômetro AccuPyc 1330 - Micromeritics.

A porosidade total foi calculada pela equação:

$$
\mathrm{P}_{\text {total }}=\left(1-\rho_{\text {relativa }}\right) \times 100
$$

A distribuição de tamanho de poros foi avaliada no porosimetro de $\mathrm{Hg}$ (Aminco 5000RG).

\section{RESULTADOS E DISCUSSÃO}

Durante testes preliminares observou-se que ocorreu um colapso imediato das bolhas das espumas obtidas com os surfactantes catiônicos, assim que incorporadas à suspensão cerâmica. Essa observação foi atribuída ao fato de a alumina possuir carga superficial negativa (o dispersante DAC é aniônico), o que ocasionou a atração entre estas partículas e a superfície das bolhas. Isto causa um acúmulo de partículas na interface líquido-ar, provocando o colapso do filme de surfactante. Por este motivo considerou-se somente a ação dos surfactantes aniônicos para a execução deste trabalho.

A avaliação de um surfactante pode ser realizada através da medida do volume de espuma formada, uma vez que o aumento da concentração do agente surfactante provoca o aumento no volume de espuma até um valor próximo à 
concentração micelar crítica (CMC). Acima deste valor, o volume de espuma mantém-se praticamente constante ou aumenta lentamente até atingir um valor constante[10]. Desse modo, o valor de CMC de um surfactante é uma medida de sua eficácia como agente espumante, já que, quanto menor a CMC menor a quantidade de surfactante necessária para se atingir um volume máximo de espuma [9].

Nesse sentido mediu-se o volume da espuma gerada usando-se os dois surfactantes aniônicos estudados. A Fig. 1 apresenta o volume de espuma em função da concentração dos surfactantes aniônicos olefina sulfonada e alquil benzeno sulfonado.

Observa-se que para a menor adição de olefina sulfonada $(0,2 \mathrm{~g} / \mathrm{L})$ o volume de espuma gerado é cerca de $400 \mathrm{~mL}$. Além disso, nota-se que adições maiores deste surfactante não resulta em um aumento significativo no volume da espuma. Isto é um indicativo de que o valor CMC do olefina sulfonada deve ser menor que $0,2 \mathrm{~g} / \mathrm{L}$. Já para o alquil benzeno sulfonado verifica-se um aumento pronunciado no volume da espuma formado quando se adiciona surfactante na faixa de $0,2 \mathrm{~g} / \mathrm{L}$ a $2,0 \mathrm{~g} / \mathrm{L}$, sendo que a partir de $1,0 \mathrm{~g} / \mathrm{L}$ o volume de espuma permanece constante o que sugere um valor de CMC próximo a este.

Entretanto, para os dois tipos de surfactantes o volume da espuma gerado alcança um mesmo valor. Estas observações demonstram que a eficácia da olefina sulfonada como espumante é maior do que para o alquil benzeno sulfonado, mas a efetividade dos dois é a mesma.

Além da eficácia na geração de espuma um outro parâmetro importante para avaliar o desempenho de surfactantes como agentes espumantes é a estabilidade da espuma gerada. Por este motivo mediu-se esta estabilidade por meio da determinação do tempo

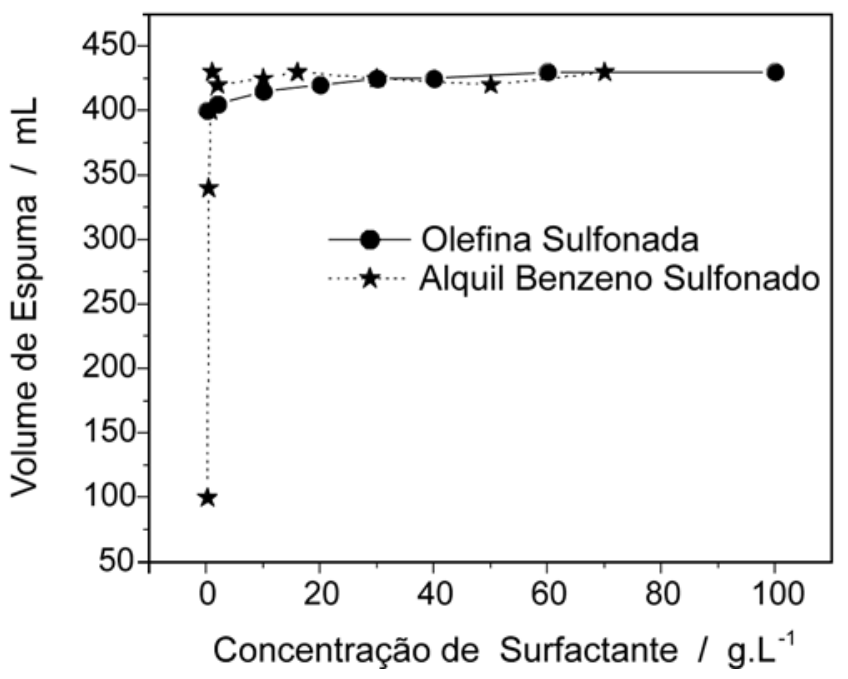

Figura 1: Volume da espuma gerada em função do teor do surfactante.

[Figure 1: Foam volume as a function of the surfactant concentration.] necessário para a drenagem da fase líquida da espuma, já que este mecanismo de degradação da espuma é o primeiro a ocorrer após sua formação [9]. A Fig. 2 mostra o gráfico representando o tempo necessário à drenagem de metade do volume inicial da solução de surfactante em função da concentração da olefina sulfonada e do alquil benzeno sulfonado.

Nota-se para o surfactante alquil benzeno sulfonado que, para concentrações maiores que a sua $\mathrm{CMC}(1,0 \mathrm{~g} / \mathrm{L})$, a estabilidade da espuma permanece constante. No entanto, para o surfactante olefina sulfonada observa-se que a estabilidade da espuma é dependente da concentração do surfactante, sendo que a estabilidade aumenta com o teor de surfactante.

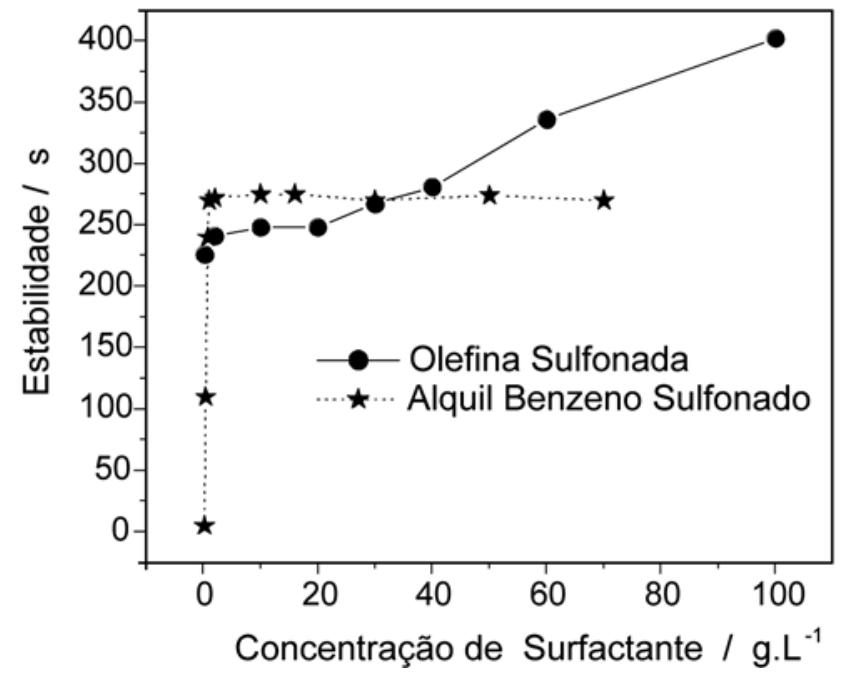

Figura 2: Estabilidade da espuma gerada em função do teor de surfactante.

[Figure 2: Stabilization of the produced foam as a function of the surfactant concentration.]

Para entender o comportamento observado para a estabilidade da espuma do surfactante olefina sulfonada é necessário considerar que esta propriedade também está relacionada às características químicas do surfactante, tais como os grupos funcionais presentes e os radicais ligados à cadeia polimérica $[7,9]$. Tais características podem ser reveladas pela análise de Infravermelho (FTIR).

Geralmente o espectro de Infravermelho apresenta a intensidade em função do número de onda $\left(\mathrm{cm}^{-1}\right)$, sendo este o recíproco do comprimento de onda. A intensidade é apresentada como porcentagem da transmitância ou da absorção de luz.

Os espectros obtidos para os surfactantes aniônicos olefina sulfonada e alquil benzeno sulfonado encontram-se na Fig. 3.

Os espectros dos surfactantes revelam bandas de absorção de infravermelho em duas regiões específicas. As bandas na faixa de $2500 \mathrm{~cm}^{-1}$ a $3500 \mathrm{~cm}^{-1}$ correspondem aos grupos 


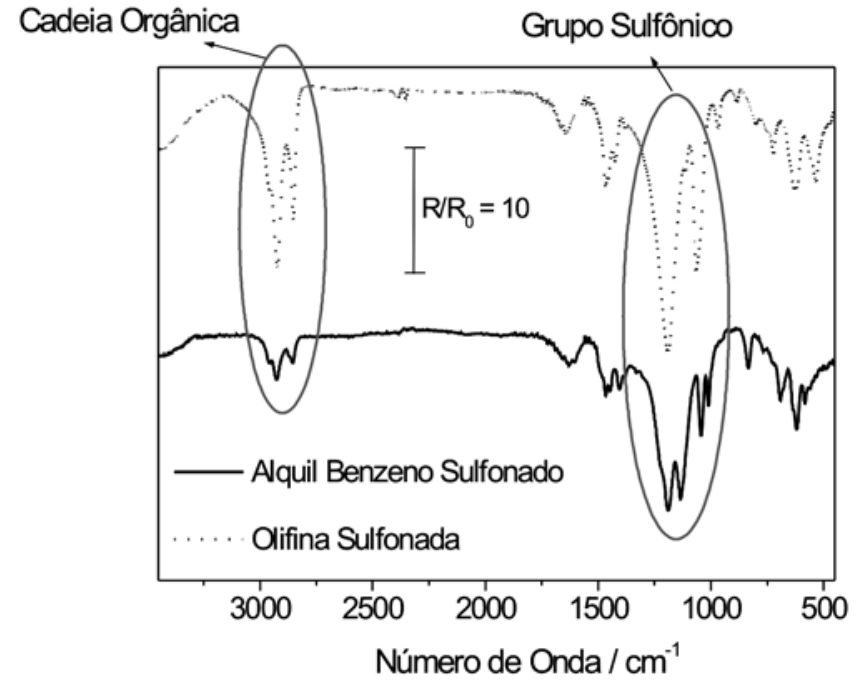

Figura 3: Espectros de infravermelho dos surfactantes olefina sulfonada e alquil benzeno sulfonado.

[Figure 3: Olefin sulfonate and alkyl phenyl sulfonate surfactants infrared spectrometry profiles.]

funcionais $(\mathrm{C}=\mathrm{O},-\mathrm{OH})$ da cadeia orgânica. $\mathrm{E}$ as bandas na faixa de $500 \mathrm{~cm}^{-1}$ a $1500 \mathrm{~cm}^{-1}$ correspondem ao grupo sulfônico, que é o radical polar ligado à cadeia orgânica.

Embora os dois surfactantes apresentem bandas de absorção nas mesmas regiões de comprimento de onda, qualitativamente pode-se observar que a razão entre as intensidades de absorção da cadeia orgânica em relação à do radical polar é maior no caso da olefina sulfonada do que para o alquil benzeno sulfônico. Consequentemente, o tamanho da cadeia orgânica da olefina sulfonada é maior que para o alquil benzeno sulfônico. Grupos orgânicos maiores favorecem a ocorrência de interações entre cadeias apolares, fato que pode explicar a maior estabilidade da espuma gerada com a olefina sulfonada.

Uma outra conseqüência do maior tamanho da cadeia apolar está no fato de que essa característica proporciona uma menor solubilidade em água, quando se comparam dois surfactantes com um mesmo grupo polar. Desta forma podese observar no espectro de infravermelho da Fig. 3 que o surfactante Olefina sulfonada possui uma solubilidade, em água, menor que o alquil benzeno sulfonado.

$\Uparrow \frac{\text { Cadeia Orgânica }}{\text { Grupo Sulfônico }} \Rightarrow \Downarrow$ Solubilidade em Água

Esta menor solubilidade é suficiente para que algumas de suas moléculas possam adsorver-se no estado sólido na interface água-ar. Este comportamento proporciona uma proteção contra a ocorrência do mecanismo de degradação de Ostwald, dificultando a difusão de gás entre esta interface [9], favorecendo a geração de espumas mais estáveis.

Com o objetivo de aumentar o tempo de estabilidade das espumas geradas com os diferentes surfactantes, adicionouse à espuma formada diferentes teores de um agente viscosificante a base de celulose. Adicionaram-se diferentes teores deste aditivo, avaliando-se sua influência no volume de espuma gerada e no seu tempo de estabilidade. Observouse que independente do teor de viscosificante não se alterou o volume de espuma, entretanto, o tempo de estabilidade aumentou significativamente. Para a produção dos corpos cerâmicos escolheu-se o menor teor de viscosificante suficiente para retardar a drenagem dos $25 \mathrm{~mL}$ da solução original por mais de $3600 \mathrm{~s}$.

Até o momento analisaram-se as diferenças químicas entre os surfactantes e seus efeitos no volume de espuma e na sua estabilidade. Porém, como estas diferenças podem afetar as propriedades finais de um corpo cerâmico poroso?

Para entender este aspecto e estabelecer correlações entre o tipo de surfactante e o processamento de cerâmicas porosas, avaliaram-se as propriedades físicas de corpos de alumina porosos produzidos com estes surfactantes.

A Tabela I apresenta os resultados de porosidade total, aparente e fechada, densidade aparente e relativa das aluminas porosas produzidas com os surfactantes olefina sulfonada e o alquil benzeno sulfonado.

Observa-se que a porosidade total final dos corpos produzidos com o surfactante olefina sulfonada foi aproximadamente 5\% superior, conferindo, desse modo, corpos porosos menos densos. Além disso, nota-se na Tabela I que para os dois surfactantes estudados uma porcentagem

Tabela I - Propriedades físicas de aluminas porosas produzidas com os surfactantes olefina sulfonada e o alquil Benzeno sulfonado e sinterizadas a $1100{ }^{\circ} \mathrm{C} / 1 \mathrm{~h}$.

[Table I - Physical properties of porous alumina produced with olefin sulfonate and alkyl phenyl sulfonate surfactants after sintering at $\left.1100^{\circ} \mathrm{C} / 1 \mathrm{~h}.\right]$

\begin{tabular}{cccccc}
\hline $\begin{array}{c}\text { Surfactante } \\
\text { utilizado }\end{array}$ & $\begin{array}{c}\text { Porosidade } \\
\text { total } \\
\mathrm{P}_{\text {total }}(\%)\end{array}$ & $\begin{array}{c}\text { Porosidade } \\
\text { aparente }\end{array}$ & $\begin{array}{c}\text { Porosidade } \\
\text { fechada } \\
\mathrm{P}_{\text {fechada }}(\%)\end{array}$ & $\begin{array}{c}\text { Densidade } \\
\text { aparente } \\
\left(\mathrm{g} / \mathrm{cm}^{3}\right)\end{array}$ & $\begin{array}{c}\text { Densidade } \\
\text { relativa }\end{array}$ \\
$\begin{array}{c}\rho_{\text {relativa }}(-) \\
\text { Olefina Sulfonada }\end{array}$ & $85,46 \pm 1,07$ & $52,79 \pm 2,85$ & $32,67 \pm 3,54$ & $0,58 \pm 0,04$ & $0,15 \pm 0,01$ \\
Alquil Benzeno Sulfonado & $81,90 \pm 0,23$ & $43,06 \pm 4,27$ & $38,84 \pm 4,39$ & $0,71 \pm 0,01$ & $0,18 \pm 0,01$ \\
\hline
\end{tabular}


significativa de poros fechados foi formada $(>30 \%)$. Tal característica estrutural é importante no direcionamento de uso destes materiais porosos, uma vez que materiais com poros fechados são mais adequados para isolamento térmico.

Além destas propriedades físicas, avaliaram-se também as distribuições de tamanho de poros das aluminas porosas produzidas com os distintos surfactantes.

Nas Figs. 4 e 5 são apresentadas as distribuições acumuladas e as distribuições diferenciais em função do diâmetro de poro para amostras de alumina porosas produzidas com olefina sulfonada e alquil benzeno sulfonado, respectivamente.

Para cada surfactante avaliaram-se 7 corpos-de-prova (cp) com o intuito de se verificar a reprodutibilidade do processo de fabricação das espumas cerâmicas. Os gráficos das Figs. 4 e 5 apresentam apenas três delas para cada surfactante.

Ao se avaliar conjuntamente os gráficos das Figs. 4 e 5 nota-
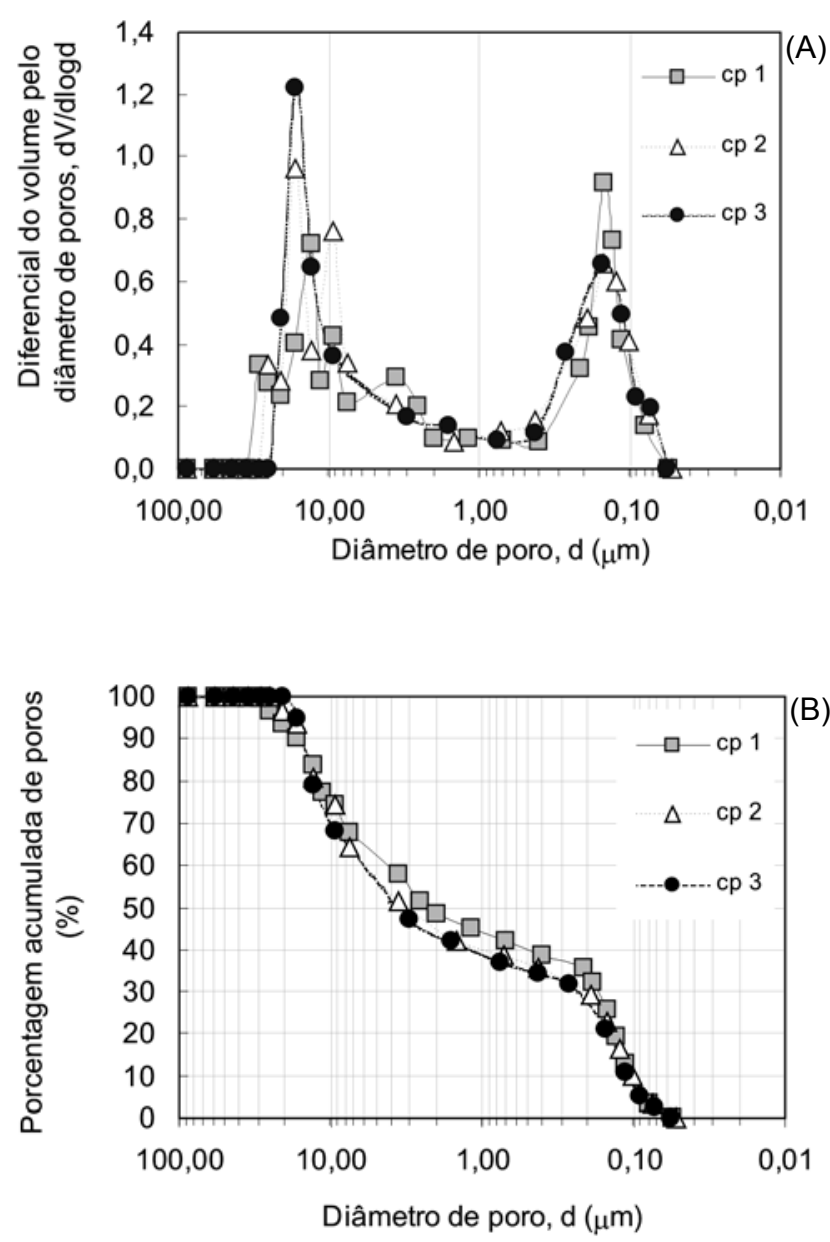

Figura 4: Distribuições de tamanho de poro (a) cumulativas e (b) diferenciais dos corpos de prova produzidos com o surfactante Olefina Sulfonada.

[Figure 4: Pore size distribution (a) cumulative and (b) differential for the samples produced with the Olefin Sulfonate surfactant.] se que a distribuição acumulada de tamanho de poros independe do tipo do surfactante utilizado, uma vez que todos os corpos de prova apresentaram $80 \%$ de poros entre $20 \mu \mathrm{m}$ e $0,1 \mu \mathrm{m}$ e diâmetro médio de poro entre $1 \mu \mathrm{m}$ e $5 \mu \mathrm{m}$.

As distribuições diferenciais indicama freqüência de diâmetro de poro presente no material cerâmico analisado. Novamente, vê-se que as amostras de aluminas porosas produzidas com os distintos surfactantes apresentaram freqüências de diâmetro de poro semelhantes. A freqüência de poros concentraram-se basicamente em duas faixas de tamanho de poros: uma faixa de $10 \mu \mathrm{m}$ a $20 \mu \mathrm{m}$ e outra na faixa de $0,1 \mu \mathrm{m}$ a $0,2 \mu \mathrm{m}$.

Tal comportamento pode ser explicado pelos processos de aeração e de incorporação da espuma à suspensão cerâmica.

Quanto ao processo de aeração, todas as espumas contêm a mesma quantidade de viscosificante e foram geradas sob mesma velocidade e tempo de mistura, consequentemente o tamanho das bolhas de ar gerado pelos dois surfactantes deve ser semelhante.
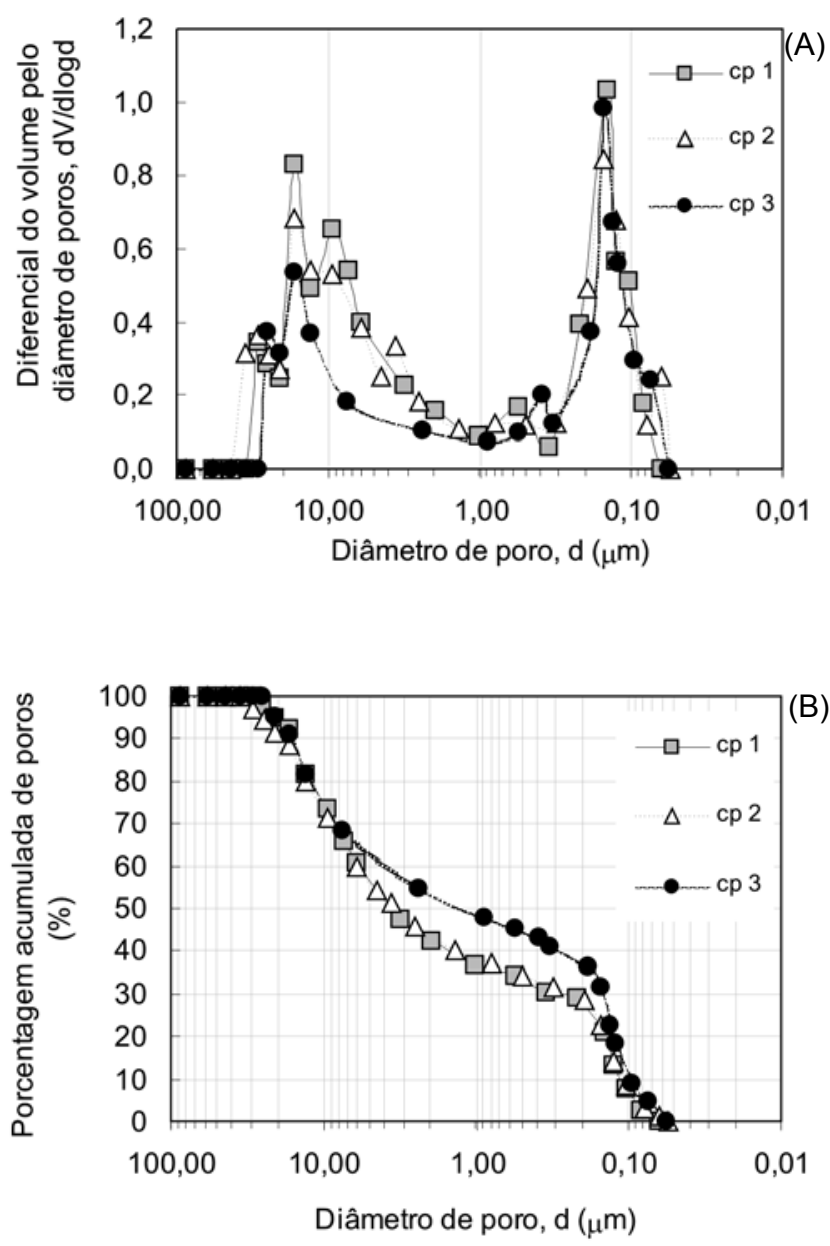

Figura 5: Distribuições de tamanho de poro (a) cumulativas e (b) diferenciais dos corpos de prova (cp) produzidos com o surfactante alquil benzeno sulfonado.

[Figure 5: Pore size distribution (a) cumulative and (b) differential for the samples produced with the alkyl phenyl sulfonate surfactant.] 
Já o processo de incorporação da espuma à suspensão parece ser de grande importância na produção das cerâmicas porosas. Estudos efetuados pelos autores têm mostrado que dependendo do método de incorporação é possível a obtenção de corpos com elevada porosidade $(90 \%)$ e população de poros predominantemente pequenos.

Desse modo, pode-se concluir que o ajuste da distribuição do tamanho de poros no corpo cerâmico final pode ser efetuado através do controle dos processos de aeração da espuma e de incorporação da mesma à suspensão cerâmica, enquanto o tipo de surfactante pode ser selecionado através da sua cadeia orgânica e grupo funcional.

\section{CONCLUSÕES}

De acordo com os resultados apresentados neste trabalho pode-se concluir que a seleção do tipo de surfactante a ser adotado para a fabricação de cerâmicas porosas deve compreender a análise de sua eficiência como agente espumante e sua estabilidade em função do tempo. Estas características irão influenciar a porosidade final dos corpos cerâmicos, uma vez que esta propriedade depende do volume de espuma adicionado à suspensão cerâmica.

Os resultados deste trabalho indicaram que o surfactante aniônico olefina sulfonada é mais eficiente e mais estável para a produção de espumas aquosas. Contudo, a distribuição de tamanho dos poros formada no corpo cerâmico parece ser dependente dos processos de aeração para formação da espuma e de sua incorporação à suspensão cerâmica.

\section{AGRADECIMENTOS}

Os autores deste trabalho agradecem a Almatis (EUA), a SKW (Alemanha) e a Fapesp pelo apoio dado a realização do mesmo.

\section{REFERÊNCIAS}

[1] T. M. Freyman, I. V. Yannas, L. J. Gibson, Prog. Mater. Sci. 46 (2001) 273.

[2] V. R. Salvini, M. D. M. Innocentini, V. C. Pandolfelli, Am. Ceram. Soc. Bull. 79, 5 (2000) 49.

[3] J. Sach, H. Seifert, DKG 76, 9 (1999) 23.

[4] J. Saggio-Woyansky, C. E. Scott, Am. Ceram. Soc. Bull. 71, 11 (1992) 1674.

[5] F. S. Ortega, P. Sepúlveda, V. C. Pandolfelli, J. Eur. Ceram. Soc. 22, 9-10 (2002) 1395.

[6] V. R. Salvini, F. Valenzuela, A. R. Studart, V. C. Pandolfelli, Anais do $48^{\circ}$ Congresso Brasileiro de Cerâmica (2004).

[7] C. D. Welker, M. A. Welker, M. F. Welker, M. A. Justman, R. S. Hendricksen, US Patent 6,153,005 (2000).

[8] J. L. Salager, "Surfactantes en Solucion Acuosa", Cuaderno FIRP S201-A, Mérida (1998).

[9] M. J. Rosen, "Surfactants and Interfacial Phenomena", $2^{\text {nd }}$ Ed., ch. 7 (1989).

[10] J. L. Salager, J. M. Anderez, A. Forgiarini, "Influencia de la Formulacion sobre las Espumas", Cuaderno FIRP 263-A, Mérida (2003).

(Rec. 27/05/2005, Ac. 21/10/2005) 